\title{
Superexponential droplet fractalization as a hierarchical formation of dissipative compactons
}

\author{
Sergey Shklyaev, ${ }^{1,2}$ Arthur V. Straube, ${ }^{3}$ and Arkady Pikovsky ${ }^{4}$ \\ ${ }^{1}$ Department of Chemical Engineering, California Institute of Technology, Pasadena, California 91125, USA \\ ${ }^{2}$ Institute of Continuous Media Mechanics, UB RAS, 1 Ac. Korolev St., Perm 614013, Russia \\ ${ }^{3}$ Department of Physics, Humboldt University of Berlin, Newtonstrasse 15, D-12489 Berlin, Germany \\ ${ }^{4}$ Department of Physics and Astronomy, University of Potsdam, Karl-Liebknecht-Strasse 24/25, D-14476 Potsdam, Germany
}

(Received 27 January 2010; published 12 August 2010)

\begin{abstract}
We study the dynamics of a thin film over a substrate heated from below in a framework of a strongly nonlinear one-dimensional Cahn-Hilliard equation. The evolution leads to a fractalization into smaller and smaller scales. We demonstrate that a primitive element in the appearing hierarchical structure is a dissipative compacton. Both direct simulations and the analysis of a self-similar solution show that the compactons appear at superexponentially decreasing scales, which means vanishing dimension of the fractal.
\end{abstract}

DOI: 10.1103/PhysRevE.82.020601

PACS number(s): 68.15.+e, 47.53.+n, 05.45.Df, 47.20.Dr

\section{INTRODUCTION}

A vast number of intriguing pattern-formation phenomena can be described with equations of Cahn-Hilliard type. Since their introduction [1], these equations have been successfully applied to a great variety of natural and technological processes such as phase separation (see, e.g., a survey [2]), topology transitions in a Hele-Shaw cell [3], dynamics of layered systems [4,5], thin films [6], population dynamics in biology [7]. In the thin film context, numerical studies of an amplitude equation of Cahn-Hilliard type [8,9] have evidenced film rupture leading to the formation of a cascade of "drops" and "fractal-like fingering" [10] comprising the gaps or "dry spots" [9] between the drops. These findings have been supported by direct simulations of the Navier-Stokes equations [11,12].

The goal of this paper is twofold. First, we relate liquid droplets with zero contact angle to dissipative compactons. A usual compacton is a well-known compact (i.e., with finite support) traveling-wave solution, which emerges in conservative systems with nonlinear dispersion [13]. We show that its stationary analog with compact support naturally appears in dissipative systems with nonlinear dissipation and, therefore, can be referred to as a "dissipative compacton" (DC). Second, we demonstrate that a DC is a primitive element mediating the formation of hierarchical fractal structure and characterize the fractal properties of this structure quantitatively.

Our basic model is a generalized one-dimensional CahnHilliard equation describing dissipative evolution of a conserved field $h(x, t)$

$$
h_{t}+\left(h^{\mu-1} h_{x}+h^{\mu} h_{x x x}\right)_{x}=0, \quad \mu \geq 3 .
$$

The flux here consists of two nonlinear terms, the first one responsible for instability of a uniform field, and the second one for the saturation of this instability. The relation between nonlinearities of these terms ensures the existence of compact self-affine solutions. Noteworthy, under certain conditions this relation describes the dynamics of a thin film over a substrate heated from below. In this example, $h$ plays the role of the local film thickness and is governed by (see, e.g., Eq. (4) in Ref. [8]):

$$
\begin{gathered}
h_{t}+\left[f(h) h_{x}+g(h) h_{x x x}\right]_{x}=0, \\
f=-\operatorname{Bo} h^{3}+\frac{B M h^{2}}{2(1+B h)^{2}}, \quad g=h^{3} .
\end{gathered}
$$

Dimensionless Bond (Bo), Biot $(B)$, and Marangoni $(M)$ numbers determine the levels of the gravity, of the heat flux at the free surface, and of the convective flow, respectively. Although function $f$ here has a rather complex form, for $h$ $\rightarrow 0$ one can set $f \approx 0.5 B M h^{2}$. This approximation holds also for moderate values of $h$, provided the gravity can be neglected, Bo $\rightarrow 0$, the heat transfer at the free surface is poor $(B$ small) while the thermocapillary effect is strong ( $M$ large). Assuming the limiting form of $f$, after an appropriate rescaling of the time, the field, and the coordinate we arrive at our basic Eq. (1) with $\mu=3$. Before proceeding to its analysis, we notice invariance under the scaling

$$
h \rightarrow p^{2} h, \quad x \rightarrow p x, \quad t \rightarrow p^{-2(\mu-2)} t,
$$

meaning that solutions with smaller $h$ evolve slower.

\section{STEADY STATE AND ITS STABILITY}

Consider non-negative stationary solutions $h=H(x)$ of Eq. (1). Looking for symmetric patterns, after one integration we obtain

$$
H H^{\prime \prime \prime}+H^{\prime}=0
$$

with a compact $H(x)$ in the form of a DC or a "touchdown steady state" [14], nonvanishing for $|x| \leq l$ only:

$$
x= \pm \sqrt{\pi \mathcal{H}} \operatorname{erf}\left(\sqrt{\frac{1}{2} \ln \frac{\mathcal{H}}{H}}\right), \quad \mathcal{H}=\max _{x} H(x),
$$

where $\operatorname{erf}(z)=\sqrt{2 / \pi} \int_{0}^{z} e^{-t^{2}} d t$. The fact of compactness makes the DC highly nontrivial, as at $x= \pm l$ the higher derivatives of $H(x)$ become singular, which can present a serious difficulty for conventional numerical approaches. Despite such a non-smoothness, exactly this solution evolves from initial data. Equation (6) presents a self-affine one-parameter family of DCs parametrized by $\mathcal{H}$ and expressed in terms of the base DC $\widetilde{H}(x)$ having $\mathcal{H}=1$ (Fig. 1): 


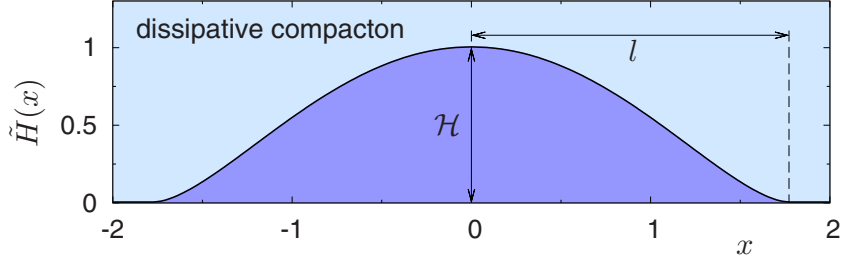

FIG. 1. (Color online) The shape of the base DC, $\tilde{H}(x)$.

$$
H(x)=\mathcal{H} \tilde{H}(x / \sqrt{\mathcal{H}}), \quad l=\sqrt{\pi \mathcal{H}} .
$$

For a thin film, the DC describes the stationary profile of a drop with the height $\mathcal{H}$ and zero contact angle. The property of self-affinity is a necessary prerequisite for the emergence of fractal structure of droplets described by Eq. (1), as we discuss below. Particularly, Eq. (7) shows that DCs become narrower for smaller amplitudes-contrary to other examples of compact solutions where typically the width and the amplitude are independent $[4,13]$.

To explore the stability of a DC, we introduce a small perturbation $\propto \xi(x) \exp (\lambda t)$ of $H(x)$, where $\lambda$ is the growth rate. By linearizing Eq. (1), we obtain

$$
\lambda \xi+\left[H^{\mu}\left(\xi^{\prime \prime}+H^{-1} \xi\right)^{\prime}\right]^{\prime}=0 .
$$

Assuming $\xi( \pm l)=0$, we multiply Eq. (8) by $\xi^{\prime \prime}+H^{-1} \xi$ and integrate by parts to arrive at an integral relation

$$
\lambda \int_{-l}^{l}\left(\xi^{\prime}-\frac{H^{\prime \prime}}{H^{\prime}} \xi\right)^{2} d x=-\int_{-l}^{l} H^{\mu}\left[\left(\xi^{\prime \prime}+\frac{\xi}{H}\right)^{\prime}\right]^{2} d x,
$$

which is closely related to the variational principle for Eq. (2) [15] and the fact that the Lyapunov functional has a local minimum on the DC. As $H \geq 0$, both the integrals in Eq. (9) are non-negative and the perturbations are nongrowing, $\lambda$ $\leq 0$. This result, however, does not guarantee against the instability, as there exist two modes of neutral linear stability, $\lambda=0$, satisfying $\xi( \pm l)=0$. One mode, $\xi_{1}^{(0)}=H^{\prime}$, reflects translational invariance of Eq. (1) and cannot give rise to instability. Another mode, $\xi_{2}^{(0)}=H-x H^{\prime} / 2$, is characterized by a nonzero volume. Our numerical simulations of Eq. (1) show that the corresponding perturbation grows nonlinearly leading to a breakup of the DC with the emergence of a complex structure. Note that for finite-amplitude perturbations of zero volume we detect the temporal decay.

\section{EVOLUTIONARY PROBLEM}

We now demonstrate numerically the formation of a fractal, hierarchical structure of DCs (see Fig. 2) for a thin film application, $\mu=3$. We discretize Eq. (1) in the domain 0 $\leq x \leq d$ with 1000 nodes, impose periodic boundary conditions, and apply the Newton-Kantorovich method [8]. We choose a distorted uniform profile $h(x, t=0)=1$ $+0.1 \cos (2 \pi x / d)$ as an initial condition. Results of computations are presented in Fig. 2. There we also compare the numerically obtained profile $h(x)$ having local maxima $h_{m}^{(n)}$, $n=1,2, \ldots$ with the DC profiles with $\mathcal{H}=h_{m}^{(n)}$, denoted as $\mathrm{DC}^{(n)}$. We see that the initial profile develops into a hierar-
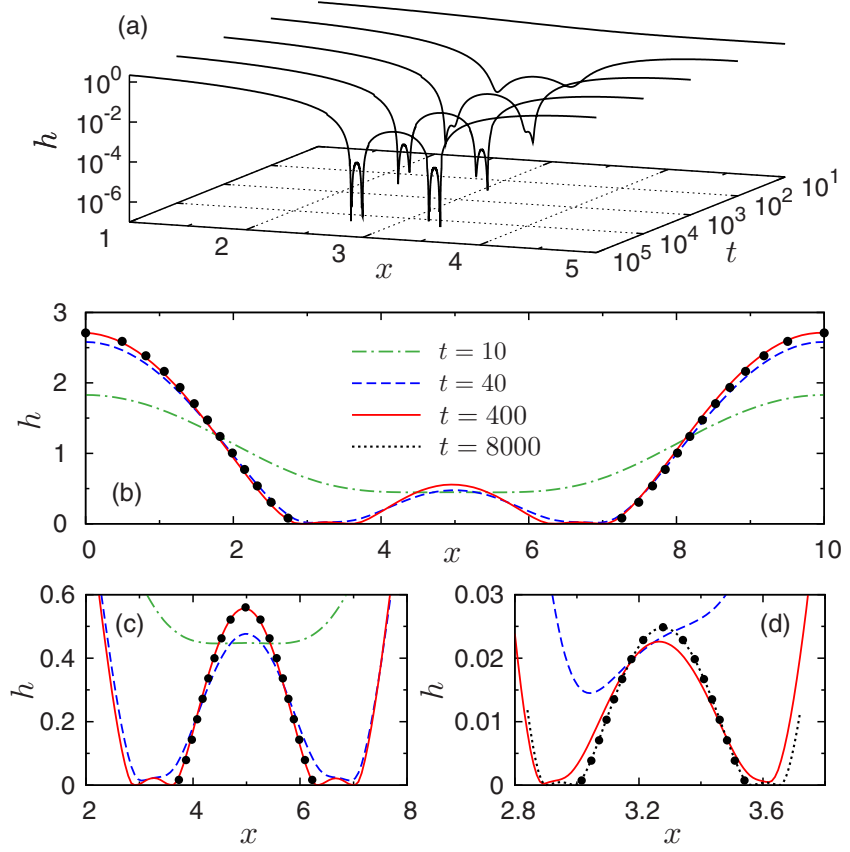

FIG. 2. (Color online) Evolution of the field $h(x, t)$ illustrating hierarchical formation of droplets, $d=10$. (a) Fragment of the evolution. Notice logarithmic scales of $t$ and $h$. (b) Snapshots of $h$. Panels (c) and (d) are zoomed in fragments of panel (b). Lines represent numerical results for Eq. (1), circles show the profiles of corresponding DCs as in Eq. (6).

chical structure of DCs of different amplitudes.

This finding is a key for resolving the structure at finer scales, as we can increase the efficiency of the numerics significantly: because after their formation the DCs remain constant in their bulk, we exclude these domains from numerical simulations and impose the corresponding boundary conditions for the still evolving domains between the formed DCs via Eq. (6). Thus, while proceeding to smaller DCs, we can considerably refine the mesh and also increase the time step, which allows us to reliably resolve high-order DCs up to $n=4$.

The observed structure along with the property of selfaffinity suggests that the formation of higher-order DCs never stops and the dry spots between DCs, form a fractal reminiscent of the Cantor set. Thus, a DC plays a role of an "intrinsic mode," inherent in the fractalization. To characterize properties of this fractal quantitatively, we plot in Fig. 3(a) the variation of $L_{n}$, the distance between the neighboring DCs of $n$th and $(n-1)$ th orders, versus the base $2 l_{n}$ of $\mathrm{DC}^{(n)}$. The numerical results for different $d$ fit well a power law:

$$
L_{n} \approx \alpha\left(2 l_{n}\right)^{\beta}, \quad \alpha \approx 0.2, \quad \beta \approx 1.25 .
$$

Note that deviations from this law for the points related to the biggest DCs stem from the initial condition. On the other hand, for higher orders the self-similarity of the formation of DCs is evident from Fig. 3(a).

Because $\beta>1$ in Eq. (10), with the increase in $n$ the ratio $L_{n} / l_{n}$ diminishes implying that the smaller daughter DCs tend to occupy the whole space between their bigger parent DCs. The fraction of dry spots tends to zero and, therefore, 

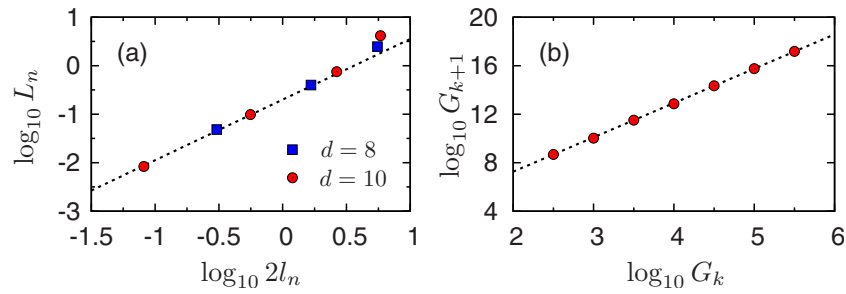

FIG. 3. (Color online) (a) The distance $L_{n}$ between two neighboring $\mathrm{DC}^{(n)}$ and $\mathrm{DC}^{(n-1)}$ versus the base $2 l_{n}$ of $\mathrm{DC}^{(n)}$. Squares and circles are numerical results for $d=8$ and $d=10$. Dotted line is a fit, Eq. (10). (b) Mapping $\log G_{k+1}\left(\log G_{k}\right)$ calculated in the framework of Eq. (14) (circles); dotted line corresponds to the asymptotic law, Eq. (15).

the fractal dimension of this set equals zero. Furthermore, for large $n$ we can neglect the distance between $\mathrm{DC}^{(n)}$ and DC ${ }^{(n+1)}$ and put $L_{n} \approx 2 l_{n+1}$. As a result, Eq. (10) entails a remarkable superexponential scaling of $l_{n}$ with $n$ :

$$
\log \left(l_{n}\right) \propto \beta^{n} \log \left(l_{0}\right) .
$$

\section{SELF-SIMILAR SOLUTION}

To alternatively support the conclusions about the fractal dimension and the superexponential scaling, we construct self-similar solutions, which originate from the rescaling property, Eq. (4). By seeking the solution of Eq. (1) in the form

$$
h=t^{-1} G(\eta), \quad \eta=x \sqrt{t},
$$

we arrive at an ordinary differential equation for $G(\eta)$ :

$$
\eta G^{\prime}-2 G+2\left(G^{2} G^{\prime}+G^{3} G^{\prime \prime \prime}\right)^{\prime}=0,
$$

where primes stand for $d / d \eta$. Numerical solutions of Eq. (13) with various initial conditions all demonstrate a qualitatively similar behavior of $G(\eta)$, which displays an infinite number of oscillations of increasing amplitudes. Two solutions with different initial conditions are shown in Fig. 4. We note that the fractal structure can be considered formed when

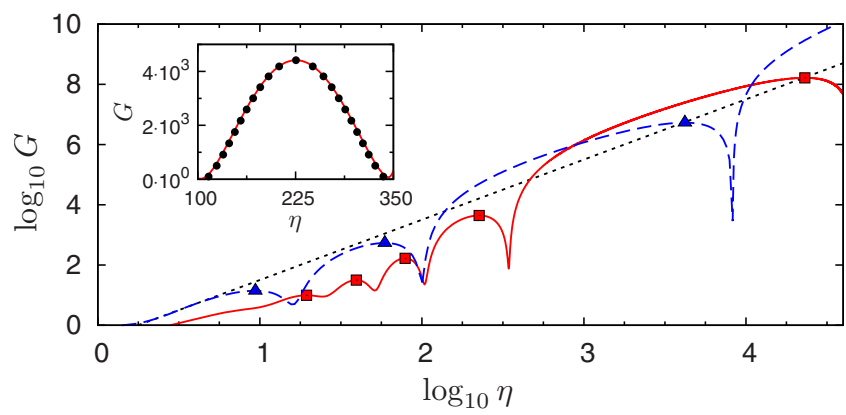

FIG. 4. (Color online) Numerical solution $G(\eta)$ of Eq. (13). Initial conditions at small $\eta$ are $G \approx 0.5 \eta^{2}$ (solid line) and $G \approx 1$ $-0.1 \eta^{2}$ (dashed line). Squares and triangles show the local maxima $G_{k}$ of $G(\eta)$; for large $k$, the maxima $G_{k}$ approach the law $G$ $=\eta^{2} / \pi$ (dotted line). Inset: a comparison of a piece of $G(\eta)$ with a single DC (circles), Eq. (6). $t$ (and, therefore, $\eta$ ) is large. In this limit, the scaling is independent of initial conditions.

For large $G$, where we can estimate $d / d \eta \sim \epsilon^{1 / 2}$ with $\epsilon$ $\sim G^{-1} \ll 1$, the first two terms in Eq. (13) become negligible in comparison with the last two terms. In this limit, Eq. (13) is reduced to Eq. (5) with $G(\eta)$ instead of $H(x)$. Therefore, $G(\eta)$ can be approximated by the solution for a DC [see Eq. (6) and the inset in Fig. 4] everywhere except for its tails, where $G$ is no more large. Thus, $G(\eta)$ looks like a sequence of DCs with superexponentially growing amplitudes $G_{k}$ $\sim \exp \left[A^{k}\right]$ and widths $\Delta \eta_{k}=2 \sqrt{\pi G_{k}}$. The positions $\eta_{k}$ of maxima for large $k$ satisfy $\eta_{k} \approx \sqrt{\pi G_{k}}$, implying the same asymptote for $G_{k}\left(\eta_{k}\right)$ for all initial conditions, see markers in Fig. 4.

To specify the superexponential growth of $G_{k}$ with $k$, we construct a mapping $G_{k} \rightarrow G_{k+1}$ valid for large $G$. In the range of $\left|\eta-\eta_{k}\right|<\sqrt{\pi G_{k}}, \quad G \approx G_{k} \tilde{H}\left(x_{k}\right)$ with $x_{k} \equiv(\eta$ $\left.-\eta_{k}\right) / \sqrt{G_{k}}$. To bridge the solution for $\mathrm{DC}^{(k)}$ with the similar one for $\mathrm{DC}^{(k+1)}$, we substitute a representation $G=\varepsilon^{-2} \zeta(y)$, $y=\left(\eta-\eta_{k}-\sqrt{\pi G_{k}}\right) \varepsilon, \varepsilon \equiv G_{k}^{-1 / 6}$ into Eq. (13) and neglect the terms $\propto \varepsilon$, which yields

$$
y_{0} \zeta^{\prime}+2\left(\zeta^{2} \zeta^{\prime}+\zeta^{3} \zeta^{\prime \prime \prime}\right)^{\prime}=0 .
$$

Here, primes denote $d / d y$ and $y_{0}=\eta_{k} / \sqrt{G_{k}}+\sqrt{\pi} \approx 2 \sqrt{\pi}$. We solved Eq. (14) numerically matching the solution to DCs at $y \rightarrow \pm \infty$, which gives the transformation $G_{k} \rightarrow G_{k+1}$ [see Fig. 3(b)]. The results fit well a power law

$$
G_{k+1} \approx 40 G_{k}^{2.83} \text {. }
$$

Equation (15) shows a superexponential growth for $G_{k}$ with $k$, as required by the self-affinity and the similar behavior for the lengths, see Eq. (11). The exponent 2.83 in Eq. (15) is in reasonable agreement with $2 \beta$ in Eq. (10), obtained within the evolutionary problem. [Recall that the amplitude $\propto l^{2}$, cf. Eq. (7)]. The fact that the correspondence is not perfect is not surprising as Eq. (15) is the asymptote of extremely large $t$ (i.e., large $k$ ), while Eq. (10) is a fit obtained for the early stage of the evolution (small $k$ ). Nevertheless, we see that the self-similar solution is closely related to the hierarchical structure of DCs described by the evolutionary problem.

We stress that the relation between the self-similar solution and the spatially periodic solution as in Fig. 2 is not simple. The whole structure of successive DC-like solutions, $h(x, t)$, obtained via Eq. (13), moves with the time toward the point $x=0$, whereas DCs $H(x)$, which are born as a result of evolution according to Eq. (1), are stationary objects. However, the long-time evolutions of both these solutions show the similar displacement of the gaps between DCs by higherorder DCs. This argument becomes transparent, if we observe the self-similar solution "stroboscopically." Let us consider a self-similar solution at moments of time $t_{k}=\eta_{k}^{2} / x_{0}^{2}$. The corresponding field profile Eq. (12) describes the formation of DCs up to the $k$ th order in the domain $0 \leq x<x_{0}$ $+\Delta \eta_{k} / \sqrt{t_{k}}$ with $\mathrm{DC}^{(k)}$ centered at $x=x_{0}$. As the growth of $G_{k}$ with $k$ is superexponential, the highest-order DC dominates the pattern, which ensures that the fractal made of the dry spots has zero dimension. 


\section{CONCLUSION}

We have applied the concept of DCs to the evolution of a thin film within a framework of the generalized onedimensional Cahn-Hilliard equation. We have shown that as a result of instability, the thin film evolves into a hierarchical structure of droplets. The fact that all the droplets can be represented by the DCs of different amplitudes is a key point in understanding the fractalization process. The knowledge of the DC properties, as an intrinsic mode of the structure, allows us to solve the long standing problem of fractal-like fingering [10]. We show that the structure is indeed a fractal, the elements of which are characterized by superexponentially decreasing amplitudes and lengths. The complementary set built of gaps between the DCs (dry spots) has zero dimension. Noteworthy, in contrast to widespread random fractals, the revealed fractal is a regular one. In this way it is similar to a recent example of an exact, soliton-based fractal in nonlinear optics [16], but with a compact basic mode.

We stress that the DC is in many ways similar to the conservative compacton (such as dissipative solitons that share many properties with conservative solitons). In both cases, an initial profile splits into a hierarchy of self-similar objects: traveling compactions [13] or stationary DCs. In the latter case the DCs arrange themselves forming a fractal. We expect this property not only for a class of Cahn-Hilliard Eq. (1) considered, but for general models possessing self-affine DCs.

Finally, we emphasize that for a thin film, DC has the meaning of a sessile droplet with the zero contact angle.
Physically, its nontrivial property of self-affinity, Eq. (7), is ensured by the existence of a flow in the droplet, cf. Ref. [4]. This link can serve as a motivation to observe a compacton and to test our predictions experimentally. Of course, fractalization does not occur ad infinitum in a real system. In our model, Eq. (1), we have neglected three factors: intermolecular (e.g., van der Waals) interaction between liquid and solid, evaporation, and gravity, which impose restrictions on the thin-film thickness $h$. For instance, for water, the role of the first two factors, where evaporation typically dominates, can be neglected at $h \geq h_{\min } \approx 10^{-3} \mathrm{~cm}$. Gravity imposes a restriction from above, $h \leq h_{\max } \approx 0.1 \mathrm{~cm}$. As a result, for a water layer of initial height $0.1 \mathrm{~cm}$ at least three generations of droplets are expected as they fit into the range $h_{\min }<h$ $<h_{\max }$. This range becomes even wider, if working with a two-layer system, as e.g., in Ref. [5]. There, evaporation is less important and we estimate $h_{\min } \sim 10^{-5}-10^{-4} \mathrm{~cm}$, which admits one or two more generations of droplets.

\section{ACKNOWLEDGMENTS}

We are grateful to A. Nepomnyashchy, A. Oron, M. Zaks, Ph. Rosenau, D. Lyubimov, and D. Goldobin for stimulating discussions. The research was supported by German Science Foundation (DFG Projects No. 436 RUS113/977/0-1 and No. STR 1021/1-2) and Russian Foundation for Basic Research (Project No. 08-01-91959).
[1] J. W. Cahn and J. E. Hilliard, J. Chem. Phys. 28, 258 (1958).

[2] A. Novick-Cohen and L. A. Segel, Physica D 10, 277 (1984).

[3] R. E. Goldstein, A. I. Pesci, and M. J. Shelley, Phys. Rev. Lett. 70, 3043 (1993); Phys. Fluids 10, 2701 (1998).

[4] P. S. Hammond, J. Fluid Mech. 137, 363 (1983).

[5] D. V. Lyubimov and S. V. Shklyaev, Fluid Dyn. 39, 680 (2004).

[6] A. Oron, S. H. Davis, and S. G. Bankoff, Rev. Mod. Phys. 69, 931 (1997); T. G. Myers, SIAM Rev. 40, 441 (1998); R. V. Craster and O. K. Matar, Rev. Mod. Phys. 81, 1131 (2009).

[7] D. S. Cohen and J. D. Murray, J. Math. Biol. 12, 237 (1981); M. A. Lewis, Theor Popul. Biol. 45, 277 (1994).

[8] A. Oron, Phys. Fluids 12, 1633 (2000).

[9] S. J. VanHook, M. F. Schatz, W. D. McCormick, J. B. Swift, and H. L. Swinney, Phys. Rev. Lett. 75, 4397 (1995); J. Fluid
Mech. 345, 45 (1997).

[10] L. Y. Yeo, R. V. Craster, and O. K. Matar, Phys. Rev. E 67, 056315 (2003).

[11] S. Krishnamoorthy, S. B. Ramaswamy, and S. W. Joo, Phys. Fluids 7, 2291 (1995).

[12] W. Boos and A. Thess, Phys. Fluids 11, 1484 (1999).

[13] P. Rosenau and J. M. Hyman, Phys. Rev. Lett. 70, 564 (1993); P. Rosenau, ibid. 73, 1737 (1994).

[14] R. S. Laugesen and M. C. Pugh, Eur. J. Appl. Math. 11, 293 (2000).

[15] R. S. Laugesen and M. C. Pugh, J. Differ. Equations 182, 377 (2002); L. Pismen, Patterns and Interfaces in Dissipative Dynamics (Springer, Berlin, 2006).

[16] S. Sears, M. Soljacic, M. Segev, D. Krylov, and K. Bergman, Phys. Rev. Lett. 84, 1902 (2000). 